\title{
VLBI AND INTERSTELLAR SCATTERING TESTS FOR SETI SIGNALS
}

\author{
V. Slysh \\ Astro-Space Center, Lebedev Physical Institute, USSR
}

\begin{abstract}
$\underline{\text { Summary }}$
Assuming that a coherent extraterrestrial intelligent transmission can be considered as a pointlike radio source, three tests are proposed to discriminate them against natural radio sources: The first test requires that strong scintillations be observed due to propagation through a turbulent interstellar medium. The second test assumes that the refractive interference should be present when the signals are refracted by larger scale interstellar turbulence. The third test involves very long baseline interferometry (VLBI) or space VLBI like the RADIOASTRON satellite to measure the angular size of the proposed SETI radio sources.
\end{abstract}

\section{$\underline{\text { Discussion }}$}

I. AL.MAR: Using space-VIBI looks very promising, in particular since there will be two dedicated satellites (Radioastron and VSOP) active in the second half of this decade. Nevertheless these satellites are for astrophysics and will observe extragalactic objects almost exclusively (quasar, AGN etc.). Do you think that appropriate objects for SETI search can be put on the observation program of these satellites?

V. SLYSH: Yes, there is a program committee which would accept reasonable proposals.

J. DREHER: If an ETI signal is narrowband, the group-delay methods of normal VLBI astrometry will not work. Will radio-astron allow phase-referenced VLBI measurements?

V.SLYSH: Yes, since there will be a phase-line between the ground-based and orbiting radio telescopes, The phase-referenced VLBI measurements will be employed for astrometric observations. 